\title{
CROSS-CULTURAL DIFFERENCES IN CREATIVE IDEATION: A COMPARISON BETWEEN SINGAPOREAN AND PORTUGUESE STUDENTS
}

\author{
Koronis, Georgios (1); Meurzec, Rianne Wally (1); Silva, Arlindo (1); Leite, Marco (2); Henriques, \\ Elsa (3); Yogiaman, Christine (1) \\ 1: Singapore University of Technology and Design, Singapore; 2: Department of Mechanical and \\ Industrial Engineering, Faculty of Science and Technology, Portugal; 3: Instituto Superior Técnico, \\ Universidade de Lisboa, Portugal
}

\begin{abstract}
The purpose of this work is to compare the creative outcome in the educational context of students belonging to two different cultures, namely Singaporean and Portuguese and determine whether they respond differently to the same design brief. The participants from both samples equal 121 student designers and span from 18-25 years old. Students were randomly distributed within a uniform, standard of student performance, which allowed for fair comparison between groups. Expert judges were employed to judge the creativity of concept sketches generated during a Collaborative Sketching exercise. To evaluate the creative outcome, we employed the Consensual Assessment Technique based on a rubric-based system developed in our earlier works. The analysis of variance procedure revealed no statistically significant difference between the averaged total scores of the two groups on the appropriateness measure. However, the student designers from both samples showed statistically significant differences when provided with a baseline brief in the novelty measure. In consideration of the overall creativity scores, a relatively equivalent performance is observed across the two universities.
\end{abstract}

Keywords: Creativity, Design engineering, Collaborative design

Contact:

Koronis, Georgios

Singapore University of Technology and Design

Singapore

georgios_koronis@sutd.edu.sg

Cite this article: Koronis, G., Meurzec, R.W., Silva, A., Leite, M., Henriques, E., Yogiaman, C. (2019) 'Cross-Cultural Differences in Creative Ideation: A Comparison between Singaporean and Portuguese Students', in Proceedings of the 22nd International Conference on Engineering Design (ICED19), Delft, The Netherlands, 5-8 August 2019. DOI: $10.1017 /$ dsi.2019.12 


\section{INTRODUCTION}

Creativity is central to human activity (Shai et al., 2013) and involves the production of original, potentially workable, ideas to solve a problem (Bourgeois-Bougrine et al., 2017). Yet, ideas rarely arise from void. Rather, our earlier research suggests students are inspired by various stimuli; thus concept ideas emerge from a set of conditions which they are exposed to (Kang et al., 2018). A review of the literature has shown that students with no experience in an industrial environment or prior experience in work groups struggle to generate original ideas and mostly reproduce existing concepts with minor changes (Voss 2014; De Napoli 2018). Other factors may well be responsible for influencing design outcomes, including cultural belonging, which is inconsistently considered in creativity research.

It is established that cultural contexts can inhibit or stimulate creative expression (Tomassoni et al., 2018) and it is a variable of that confluences creativity along with personal variables (Sternberg 2006). This environmental milieu is always present with a profound effect on the creative expression of individuals.

Culture generates diversity and it is naturally revealed in all human action, including the products that people design (Moalosi et al., 2010). As Morris and Leung (2010) propose; Western scholars seem to prioritize novelty over appropriateness, whereas Eastern scholars prioritize appropriateness over novelty. Eastern conception of creativity, according to (Lubart 1998) is dynamic, involving the reuse and reinterpretation of tradition rather than breaks in the tradition compared to a western definition of creativity as a product-oriented originality-based phenomenon. In a psychological paper, Nisbett and Masuda (2003) highlight that East Asians and Americans have broadly different cognitive styles.

According to Hofstede (2001), whose scholarship studies differences between cultures, countries that score highly on the uncertainty avoidance index (UAI), defined as the extent to which members of a culture feel threatened by ambiguity, and long-term orientation (LTO), defined as the fostering of virtues oriented towards future rewards as opposed to tradition, have notable differences (Hofstede and Minkov 2005). Hofstede's framework infers that countries exhibiting high UAI (such as Portugal) maintain a rigid code of beliefs and behaviors, are intolerant of unorthodox behaviors and ideas and may resist innovation. Portugal also scores lowly on LTO, indicating a preference for normative thought, over pragmatism, and an avoidance for uncertainty to achieve quick results. Singapore, conversely, ranks in the last place for UAI but scores highly on LTO, thus demonstrating that this culture places importance in long-term investments such as perseverance and achieving results over a period of time and cultural qualities supporting long-term investment, such as perseverance.

Gautam and Blessing (2009) argue in their study that the approaches of designers are influenced by their cultural background. Accordingly, we sought to explore whether cultural differences influence creative outcomes in an ideation session by evaluating and comparing students' responses to a design task. This paper sought to contribute to the discussion of cross-cultural ideation by considering two populations, namely Singaporean and Portuguese samples. Previous efforts to quantify cultural differences have been investigated (Vaughan et al., 2013), however, failed to compare the design problems across cultural settings, thus our research sought to provide such a comparative simulation in our cultural samples. Singapore students, in the sample, were undergraduates, while their Portuguese counterparts were graduates. This paper reviewed the results from Collaborative sketching (C-Sketch) ideation method we tested among student designers from two different cultural environments to identify differences in creative outcomes. We constructed the following research questions to assess this:

1. For design outcomes: Are there any statistically significant differences in the creative outcome to be found between culturally diverse students' scores?

2. For the design briefs: Do any of the design briefs result in mean creativity scores that do not differ significantly between the two cultural samples?

\section{EXPERIMENTAL METHOD}

This experiment assesses the statistically significant differences of design outcomes across various design briefs between two cultural samples. To test for variance, we explored three experimental conditions: a baseline design brief, which is a succinct problem description; a visual condition paired with the baseline design brief, in which a video shows a user interacting with a device; and a quantivisual condition paired with the baseline design brief, in which numerical requirements for the desired product is presented with the visual condition. The above conditions are defined in section 2.1. In the two cultural 
contexts, all participants were provided with the same experimental procedure and documentation media in the English language. Each class of student designers was assigned randomly to one of the three experimental conditions and informed that their participation would have no impact on their academic grades. Students had no prior C-Sketch training and were provided with instructions to use this technique to generate ideas as an effective method for encouraging creative ideation (Shah et al., 2001). At the start of the exercise, each group of students was given an A3 sheet divided into three sections. Each student spent the first 15 minutes of the exercise sketching three separate ideas in response to the design brief. Next, the students passed their sheet of paper to the right and added inputs to their group members' ideas. All briefs were deliberately written to permit the participants to explore a broad range of concepts, so they would not be unduly penalized for lack of knowledge in any specific domain.

\subsection{Description of the design problem}

The participants in both universities were asked to design a device to extract juice from fresh oranges at home. This design problem has also been used in previous work on idea generation sessions Koronis et al. (2018). Each of the 3 classes (within each university) was given a different design brief on the theme of orange squeezer devices. The students were asked to sketch their most creative ideas and were infromed that creativity would be assessed based on novelty and appropriateness.

It was expected that participants would not have extensive prior experience in solving this problem, yet orange juice extraction devices were deemed common devices that the majority of students would have encountered before. A post-experiment survey was conducted to measure student familiarity with orange juice extraction devices in both universities. The post-experiment survey $(n=62)$ found that $9.68 \%$ of Singaporean students were fully aware of orange juicers and were regular users of orange juicers, almost $80 \%$ were aware of such devices though they were not regular users, and only $6.45 \%$ were unaware of such devices. For the Portuguese sample $(n=60) ; 35 \%$ of students were fully aware of orange juicers and were regular users of orange juicers, almost $65 \%$ were aware of such devices though they were not regular users and only $5 \%$ were unaware of such devices.

Each class was given the baseline design brief with different combinations of supplementary information in the form of visual or quantivisual stimuli. Table 1 summarizes the elements of the briefs $(a, b, c)$, where the baseline brief is referred to as (a), the visual condition that is paired with a baseline brief is referred to as $(a+b)$, and lastly the quantivisual condition paired with a baseline brief is referred to as $(a+b+c)$. The visual condition was projected to respective students in class, which showed users extracting juice manually using a conventional orange squeezer. The quantivisual design brief provided both the visual condition and detailed quantitative requirements, such as numerical requirements for product cost, the maximum number of manufacturing processes, the devices' wash-ability and product volume.

Table 1. Summary of design brief conditions

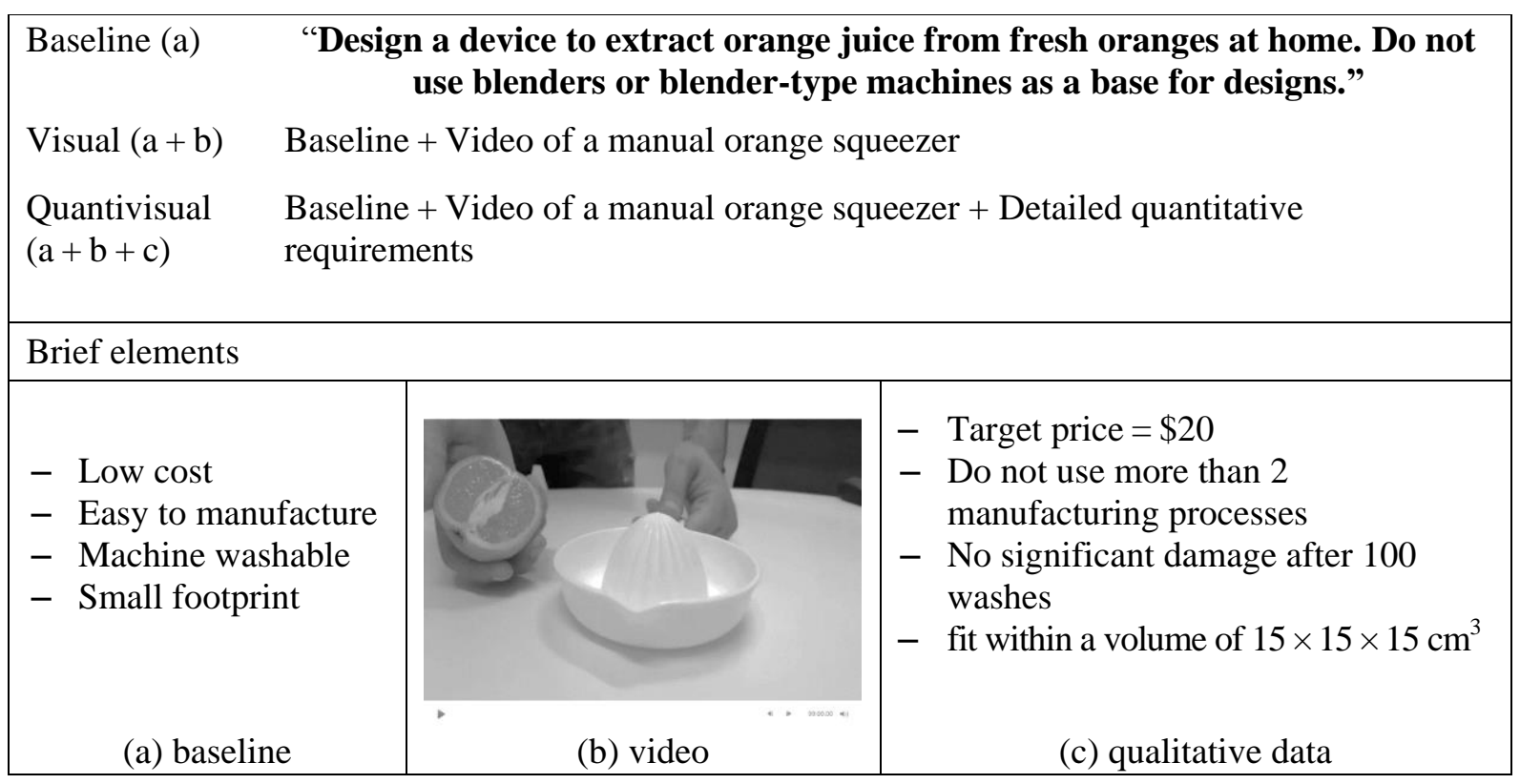




\subsection{Participants}

The total sample consisted of 121 student designers between the ages of 18 and 25, belonging to two different cultures: Singaporean $(\mathrm{N}=65)$ and Portuguese $(\mathrm{N}=56)$. The participants of the Singaporean setting were first-year undergraduate engineering/architecture student designers in an engineering course, while the Portuguese participants were graduate students in a mechanical engineering course. Table 2 shows descriptive statistics for the samples, with the mean scores and standard deviations (SD) where counts are rounded off to the nearest integer while percentages and percentage points (pp) are rounded to three significant figures (3 s.f.).

Table 2. Descriptive statistics for each sample

\begin{tabular}{|c|c|c|c|c|c|}
\hline Category & sgMean & sgSD & ptMean & ptSD & Rounding \\
\hline Sample size & 22 & 1 & 19 & 1 & Integer \\
\hline Females per class* & $8(36.9 \%)$ & 1 (4.65pp) & $5(25.1 \%)$ & 2 (11.0pp) & $\begin{array}{c}\text { Integer } \\
3 \text { s.f. }\end{array}$ \\
\hline Males per class* & $14(63.9 \%)$ & 1 (4.65pp) & $14(74.9 \%)$ & 2 (11.0pp) & $\begin{array}{c}\text { Integer } \\
3 \text { s.f. }\end{array}$ \\
\hline
\end{tabular}

The "sg" prefix indicates Singaporean data samples and "pt" the Portuguese data samples

\subsection{Creativity assessment}

Literary consensus depicts that creativity includes the features of novelty and appropriateness (Amabile 1982; Kampylis and Valtanen 2010; Madni 2012), thus we focused on those two metrics for the design outcome evaluations. Novelty is defined as the originality and 'surprising-ness' of a concept and how unusual or unexpected an idea is compared to other ideas (Shah et al., 2003) while appropriateness, or "quality", is defined as the alignment of a concept with the requirements in the design brief. Being focused on the quality (rather than quantity) of the design outcomes we did not account for the number of ideas within a sketch or the concept progression when scoring the outcomes. As such, we treated all ideas within a sketch as equally valid.

We relied on computing the creativity scores based on ratings from expert judges, namely professionals and academicians with at least 3 to 4 years of design education, and exposure to engineering design or product development. Five judges were involved in the evaluation process: two separate pairs of independent raters evaluated concept drawings from each university while one judge evaluated all the sketches from the Singapore and Portugal samples. All sketches were assessed following Amabile's (1996) peer evaluation technique utilizing a rubric-based system using a 5-point Likert scale (see Table 3). These guidelines helped the judges to standardize the evaluation of drawings for the two metrics of novelty and appropriateness. Each assortment of judges went through a training set of 6-10 drawings for familiarization with the evaluation rubric and evaluation process. Two judges went through each sketch for each sample, and one judge went through both samples wholly. Each sketch received scores from three judges, before being averaged to produce a mean score for each sketch on each of the metrics. At large, the judges valued and scored high in novelty the ideas that demonstrated divergent thinking and came from other domains even if those ideas were not feasible. Replication of existing products predisposed them to mark with the lowest grade possible ( 1 out of 5 ), even though those existing solutions could still score high in the appropriateness as those metrics are established as orthogonal.

Most participants generated 3 sketches to the design problem; however, not all sketches were evaluated and analyzed. As it often occurred in our experiment, students repeatedly nominate their first idea as their best in surveys conducted after the C-Sketch activity. Subsequent ideas observed to be repeated variations or improved versions of the initial design. Participants also failed to consistently complete three ideas in the time they were given to complete the C-Sketch activity. Our intention was to eliminate potential carryover effects from one sketch to the next in order to capture novelty and appropriateness, rather than repetitive iterations of similar concepts. To simplify sampling and ensure successful statistical analyses, this concluded our decision to explore the first sketch only. 
Table 3. Rubric for creativity evaluation

\begin{tabular}{|c|c|c|c|}
\hline Novelty & Score & Level & Example \\
\hline \multirow{5}{*}{$\begin{array}{l}\text { The extent to } \\
\text { which the design is } \\
\text { different from the } \\
\text { usual way of } \\
\text { extracting juice. }\end{array}$} & 1 & Entirely similar & Copy of existing product \\
\hline & 2 & Quite similar & Minimal differences \\
\hline & 3 & Some similarities/ & Average no surnrises \\
\hline & & differences & Minimolcimiloritios \\
\hline & 4 & Quite anterent & Mimimal simiraritues \\
\hline & & & \\
\hline Appropriateness & Score & Level & Example \\
\hline \multirow{5}{*}{$\begin{array}{l}\text { The extent to } \\
\text { which the design is } \\
\text { aligned with the } \\
\text { brief guidelines. }\end{array}$} & 1 & Not aligned & Does not meet the guidelines \\
\hline & 2 & Slightly aligned & Falls in some guidelines \\
\hline & 3 & Somewhat aligned & $50 / 50$ aligned to guidelines \\
\hline & 4 & Mostly aligned & $\begin{array}{l}\text { Meets most of the design } \\
\text { ouidelines }\end{array}$ \\
\hline & 5 & Completely aligned & Fully aligned with the brief \\
\hline
\end{tabular}

\subsection{Inter-rater reliability and ANOVA testing assumptions}

Upon completion of expert creativity judging, interrater reliability was checked for consistency between judges. The degree of agreement among judges is reported under the intra-class correlation coefficient (ICC-1) estimates in Table 4 based on a two-way random effect, average measures model. In the distributional label column the first part of the label connotes the location: "Sg" for Singapore and "Pt" for Portugal, novelty (Nov) and appropriateness (App) are also indicated. The IBM SPSS version 25 was utilized for all the calculations and ICC estimates. Based on the $95 \%$ confidence interval of the ICC estimates, all reliability values were in the good to fair range, with novelty scores $\mathrm{ICC}=0.687$ (sgNov), 0.678 (ptNov) and appropriateness scores ICC $=0.781$ (sgApp), 0.764 (ptApp). We sought to run an ANOVA to ascertain the statistical differences between conditions. First, the data was checked for ANOVA assumptions, and while the data passed the homoscedasticity, skewness and kurtosis assumptions, it failed to meet normality assumptions (Table 4). The Levene's Test for Equality of Variances did not show any departure from homogeneity $(p>0.05)$ and the assumption of homoscedasticity was tested and found to be acceptable. A Shapiro-Wilk test was employed to ascertain normal distribution across the samples, however, the null hypotheses failed to be rejected for the SgNov and PtNov dependent variables $(\mathrm{p}<0.05)$ indicating a non-normal distribution and inferring the use of non-parametric measures for analyses. A Kruskal-Wallis ANOVA was implemented instead.

Table 4. Interrater reliability results and ANOVA assumption testing

\begin{tabular}{|l|l|l|l|l|}
\hline $\begin{array}{l}\text { Distributional } \\
\text { label }\end{array}$ & $\begin{array}{l}\text { Total no. of } \\
\text { observations }\end{array}$ & $\begin{array}{l}\text { Intraclass } \\
\text { correlation }^{\mathrm{a}}\end{array}$ & $\begin{array}{l}\text { Normality test, } \\
\text { Shapiro-Wilk }\end{array}$ & $\begin{array}{l}\text { Homoscedasticity, } \\
\text { Levene's Test }\end{array}$ \\
\hline sgNov & 65 & $0.687^{\mathrm{b}}$ & nonnormal & all variances \\
\cline { 1 - 3 } ptNov & 56 & $0.678^{\mathrm{b}}$ & nonnormal & homogeneous \\
\hline sgApp & 65 & $0.781^{\mathrm{b}}$ & normal & all variances \\
\cline { 1 - 2 } & 56 & $0.764^{\mathrm{b}}$ & normal & homogeneous \\
\hline
\end{tabular}

${ }^{\mathrm{a}}$ Type $\mathrm{C}$ intraclass correlation coefficients using a consistency definition. The between-measure variance is excluded from the denominator variance.

${ }^{\mathrm{b}}$ This estimate is computed assuming the interaction effect is absent because it is not estimable otherwise.

\section{RESULTS AND DISCUSSION}

Through this experiment, 121 concept sketches were collected from the two cultural settings. The experimental layout along with the mean scores and standard deviations for each trial condition, using the measure of novelty and appropriateness is summarized in Table 5. The label header follows the naming criteria found in Table 1. 
Table 5. Design brief variations and values of dependent variables

\begin{tabular}{|c|c|c|c|c|}
\hline \multirow[t]{2}{*}{ Briefs variable } & \multirow[t]{2}{*}{ Label } & \multirow{2}{*}{$\begin{array}{l}\text { No. of } \\
\text { sketches }\end{array}$} & \multicolumn{2}{|c|}{ Dependent variables } \\
\hline & & & Novelty (SD) & Appropriateness (SD) \\
\hline Baseline & $\mathrm{bSg}$ & 22 & $3.23 \pm 0.79$ & $2.46 \pm 0.88$ \\
\hline Visual & $\mathrm{vSg}$ & 22 & $2.29 \pm 0.81$ & $3.35 \pm 0.74$ \\
\hline Quantivisual & qvSg & 21 & $2.67 \pm 0.70$ & $2.76 \pm 0.83$ \\
\hline Baseline & $\mathrm{bPt}$ & 19 & $2.35 \pm 0.78$ & $2.93 \pm 0.69$ \\
\hline Visual & $\mathrm{vPt}$ & 17 & $2.78 \pm 1.00$ & $3.18 \pm 0.87$ \\
\hline Quantivisual & qvPt & 20 & $2.75 \pm 0.97$ & $2.98 \pm 0.79$ \\
\hline Totals & & 121 & $2.68 \pm 0.88$ & $2.93 \pm 0.84$ \\
\hline
\end{tabular}

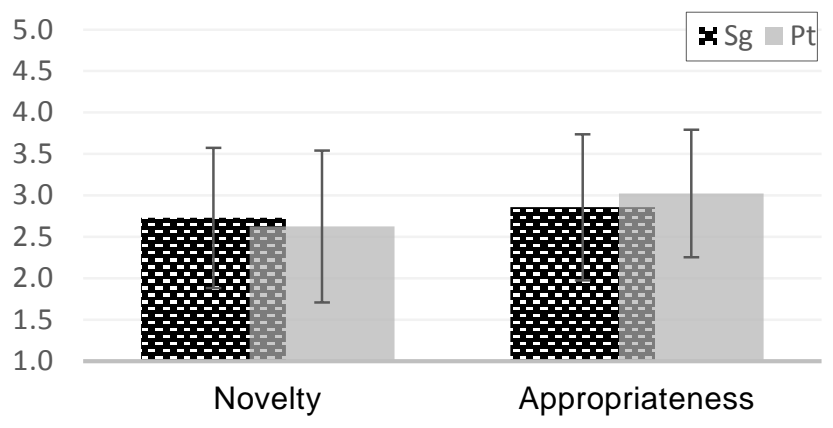

Figure 1. Overall mean creativity scores for Singaporean $(S g)$ and Portuguese (Pt) participants

\subsection{Novelty}

Novelty was the only dependent variable to illustrate significant differences between the samples, with Singaporean students in the baseline condition scoring higher than their Portuguese counterparts. The lowest scoring brief for novelty is the Singapore visual brief $(\mu=2.29)$.

Figure 2a illustrates an example of a highly novel solution, while Figure $2 b$ is an example of a copied solution that already exists, thus a low score for novelty. Figure 2a is highly novel, despite the fact that it is modifying an existing product as suggests a sophisticated machine characterized by an electric moving system fed by batteries. It is remarkable that although no additional aids or props were provided to the design team, the produced solution scored the highest possible score novelty. In Figure 1, the overall scores (all briefs under one bar) are plotted for each metric in both universities, and the means of both samples are comparably similar.
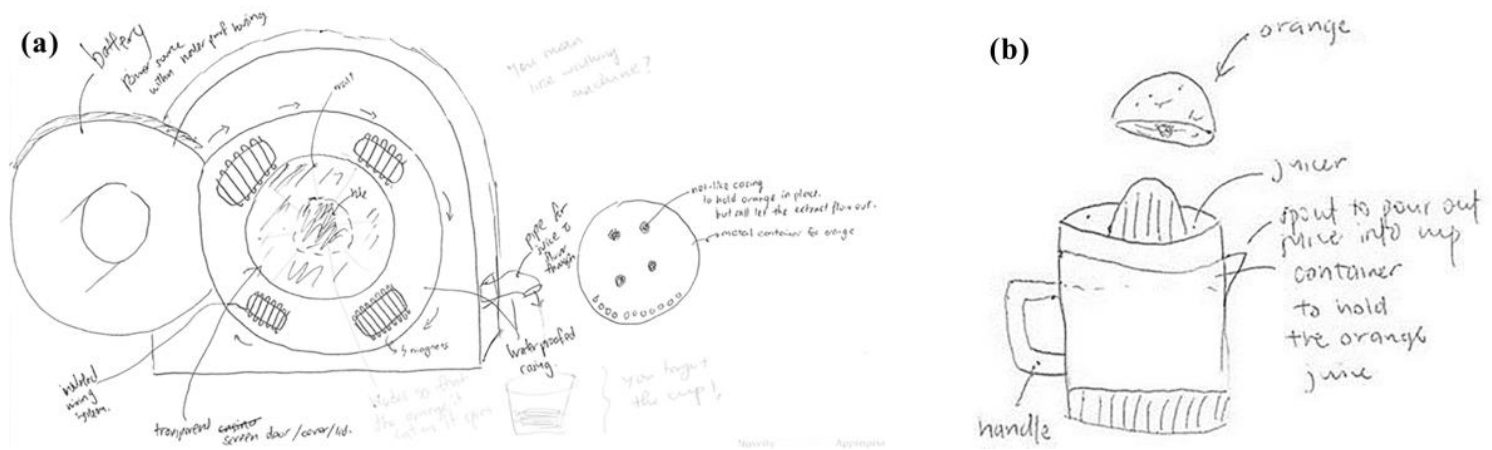

Figure 2. Sketches exposed to the baseline brief, in which (a) received a high novelty score, and (b) received a low novelty score 


\subsection{Appropriateness}

The mean appropriateness score, on the other hand, improves when quantitative requirements and visual stimulus are provided. Earlier factorial studies we published explained the need for good examples of successful products and detailed specification in the design process to ensure that students' concepts are aligned with the brief guidelines (Kang et al., 2018; Koronis et al., 2019). In the appropriateness metric, both Singaporean and Portuguese participants in the visual condition scored higher than their baseline counterparts. The lowest scoring brief for appropriateness is the baseline condition in the Singapore sample $(\mu=2.46)$. In summary, the novelty metric was more apparent in the Singapore population, while the appropriateness metric was more highly scored upon in the Portuguese sample.

Figure 3a shows a highly appropriate sketch, which responds well to the needs of the brief (e.g., easy to manufacture, washable, low cost, etc.). It is possible that the supplementary quantitative information helped students pay particular attention to these details. The video example probably guided sketches to include tangibly ways to materialize the requirements Figure $3 \mathrm{~b}$ illustrates a lowly appropriate sketch, which failed to address the design requests due to its complexity making it difficult to manufacture, clean and keep at low cost.

\section{(1)}

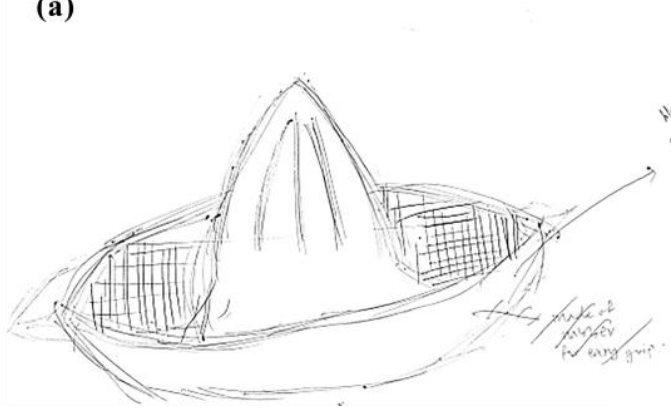

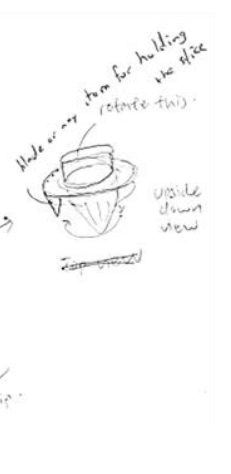

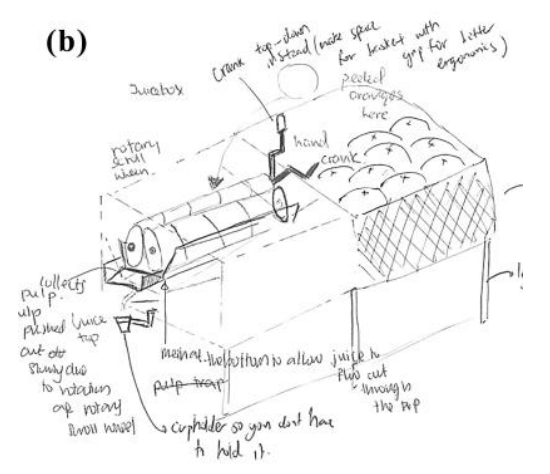

Figure 3. Sketch from the quantivisual brief that scored highly on appropriateness (a), and an example of a sketch scoring lowly on appropriateness (b)

\subsection{Statistical analysis ANOVA}

The data was non-normally distributed for the Novelty dependent variable (for the sgNov set), however, all other ANOVA assumptions were met. As such, a Kruskal-Wallis, the non-parametric equivalent test was employed.

\subsubsection{Novelty statistics}

The Kruskal-Wallis Test revealed a statistically significant difference in novelty scores across six different conditions (Gp1, $n=22$ : Baseline Singapore, Gp2, $n=22$ : Visual Singapore, Gp3, $n=21 \_$: Quantivisual Singapore, Gp4, $n=19$ : Baseline Portugal, Gp5, $n=17$ : Visual Portugal, Gp6, $n=20$ : Quantivisual Portugal), $\chi^{2}(5, n=121)=17.2, p=0.04$. Kruskal-Wallis test pairwise comparisons indeed point out an in-between difference across briefs delivered to classes of the same university (bSg - vSg, $\mathrm{p}=0.001$ ), but those are not within the scope of this paper.

\subsubsection{Appropriateness statistics}

Further, the Kruskal-Wallis Test revealed no evidence of statistical difference in appropriateness scores across three different conditions (Gp1, $n=22$ : Baseline Singapore, Gp2, $n=22$ : Visual Singapore, Gp3, $n=21 \_$: Quantivisual Singapore, Gp4, $n=19$ : Baseline Portugal, Gp5, $n=17$ : Visual Portugal, Gp6, $n=20$ : Quantivisual Portugal), $\chi^{2}(5, n=121)=13.8, p=0.017$. Again, in this comparison the pairwise Kruskal-Wallis test indicated in-between $(\mathrm{bSg}-\mathrm{vSg}, \mathrm{p}=0.011)$ which is not studied for this paper.

The simultaneous comparison intervals in Figure 4 illustrates the confidence interval (CI) for the selected pairwise comparisons between the two university samples to demonstrate the differences between the conditions for novelty and appropriateness. When an interval does not contain zero, the corresponding means are significantly different. 


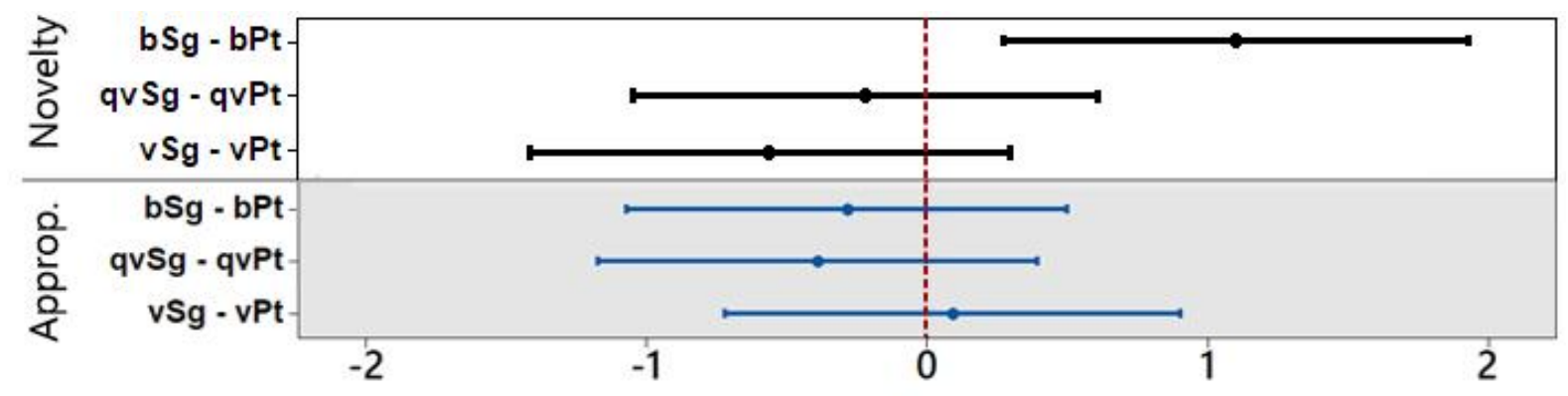

Figure 4. Simultaneous 95\% Cls for Novelty and Appropriateness, if an interval is not intersecting the dashed central line (zero), the corresponding means are significantly different, $(S g=$ Singapore, $P t=$ Portugal, $b=$ baseline, $v=$ visual, $q v=$ quantivisual)

\subsection{Discussion}

The product design process is inevitably influenced by the designer's culture (Razzaghi and Ramirez 2005); just like almost all aspects of human life are influenced by culture (Hofstede 2001). It is assumed that ideation in the early stage of the concept development which studied herein is not exempt from these pervading cultural effects. The cultural difference can also be linked to the characteristics of culture and affect the courses of a design process (Gautam and Blessing 2009). Regardless of the above suggestions, creative outcomes in our study, across two cultures, were found to be substantially similar in overall creativity, with only slight differences. Analogous results in the creativity outcomes have been reported by Tomassoni et al. (2018) when comparing the creative drawings of children in primary schools from two different cultures and found no statistically significant difference between their averaged total scores. Just like in their experiment, our study concluded that the Singaporean and Portuguese participants did not tackle the design problem in culturally divergent ways to bring up significantly different results overall.

In response to RQ1, we questioned whether differences in the creative outcome would be found between students' scores of the two different cultural contexts. We found statistically significant differences in the creative outcome between the briefs only for those in the baseline condition (bSg and bPt). Baseline briefs are short descriptions of the design problem with no additional stimuli or requirements. Those design briefs yielded sketches that rated significantly differently on novelty scores for the Singapore and Portugal samples. Interestingly, the Portuguese sample had participants of older average age and was undertaking postgraduate studies, while Singaporean students were generally of a broader age range and undertaking their undergraduate studies. The Singaporean sample also had a larger female representation in comparison to their Portuguese counterparts. The results found that Singaporean students fared better on the novelty metric in the baseline group. Studies suggest that gender in itself may not explain the differences, but rather gender roles may act as a mediating factor on creativity (Stoltzfus et al., 2011). One plausible explanation could be that the Singaporean sample was more novice in its education and perhaps, therefore, carried less domain specialization. Further, they may have fewer constraints for solutions, due to their inexperience (James et al., 2014). The reason behind the differences, however, remains speculative and would carry more weight if the differences had been seen throughout the conditions.

With respect to RQ2, we sought to investigate the brief with the least statistical difference, within the creativity scores for the culturally diverse populations. It was shown that when pairing the baseline brief with visual stimulus and numerical requirements; that brings the potential to eliminate differences between the different sample groups (Figure 4). Similar results were reported when providing students with a visual example along with the baseline brief. Therefore, it appears to be more effective to give specific requirements and examples when opting for higher average creative outcomes which do not diverge among different cultural groups of student designers (visual and quantivisual briefs). Our earlier factorial studies on larger samples, however, suggest that pairing visual with a baseline brief decreases novelty scores of sketches (Koronis et al., 2018), implying that is best to avoid providing any additional information at all. Further, we found it essential to introduce good examples of successful products and detailed specifications in the design process to ensure that students' sketches are aligned with the design brief guidelines (Kang et al., 2018). The aforementioned factorial results from our earlier studies along 
with the current pairwise comparison combine to suggest that the visual stimuli and numerical requirements paired with baseline briefs are adequate for lessening creativity outcome differences in different cultural groups.

\section{CONCLUSIONS AND LIMITATIONS}

An idea generation process was conducted with student designers of two engineering-oriented universities. The primary objective of this research was to examine the statistical differences of the creative performances in ideation between two groups of students and observe whether cultural differences impacted design outcomes. Significant differences emerged on novelty scores for recipients of the baseline design brief in Singaporean and Portuguese samples. Our study faced some limitations, notably the small samples, and heterogeneity of participants, such as their varied educational backgrounds.

Considering the overall novelty and appropriateness scores, they appear to be relatively similar for both universities' student designers. We provide valuable insights into the idea generation of student designers in Singapore and Portugal cultural context. The findings could potentially be valuable for companies with multicultural designers, to deliver briefs that are tailored to promote desired creative outcomes. In the corporate environment, experienced designers from an international firm visit an overseas branch to join other senior or junior designers and solve a problem or design a product for the global market. This could be a case scenario where all should be provided with more requirement-specific examples and visual stimuli so that they can avoid having diverged overall creativity outcomes. Moreover, hopefully, educators and project managers may also have insight into the ideal brief for culturally diverse groups.

Statistically significant differences in the design outcomes may reflect practically important differences between cultures. In both universities; all groups included students of different nationalities, especially as both universities are populated by students from nearby countries. Further data analyses involving measures of cultural effects are necessary to identify and conclusively explore the impact of culture on creativity outcomes. Additionally, the employment of university students as participants may be imperfect when considering adult and professional samples. Future research in this area should consider a more extensive study, incorporating professionals from engineering, design, and architecture to support the findings of this paper.

\section{REFERENCES}

Amabile, T.M. (1982), “The Social-Psychology of Creativity: A Consensual Assessment Technique”, Journal of Personality and Social Psychology, Vol. 43 No. 5, pp. 997-1013. http://dx.doi.org/10.1037/00223514.43.5.997

Amabile, T.M. (1996), Creativity in Context, 1st ed., Westview Press, Boulder, CO.

Bourgeois-Bougrine, S., Buisine, S., Vandendriessche, C., Glaveanu, V. and Lubart, T. (2017), "Engineering Students' Use of Creativity and Development Tools in Conceptual Product Design: What, When and How?", Thinking Skills and Creativity, Vol. 24, pp. 104-117. http://dx.doi.org/10.1016/j.tsc.2017.02.016

De Napoli, L., Rizzuti, S. and Raco, A. (2018), "How Mood Fosters Creativity in Product Design? Experimental Evidences on Humour Impact During a Conceptual Design Session in a Master Degree Class", In: Bohemia E., Kovacevic A., Buck L., Childs P., Green S., Hall A., and Dasan A. (Ed.), E\&PDE 2018, London, UK, 6-7th September, pp. 242-247.

Gautam, V. and Blessing, L. (2009), "Cultural Influences on Design Processes - An Empirical Study”, In: Norell Bergendahl M., Grimheden M., Leifer L., Skogstad P., and Lindemann U. (Ed.), International Conference on Engineering Design, Palo Alto, USA, 24-28th August, Institution of Engineering Designers, pp. 115-122.

Hofstede, G. (2001), Culture's Consequences: Comparing Values, Behaviors, Institutions and Organizations Across Nations, Tilburg University, Sage Publications, Netherlands.

Hofstede, G. and Minkov, M. (2005), Cultures and Organizations: Software of the Mind, McGraw-Hill, New York.

James, T.J.Y., Kuan, Y.K., Parveen, H., Huang, Z.X., Ganeshkumar, R., Behera, J., Sanaei, R., Otto, K. and Hölttä-Otto, K. (2014), “An Overview of Design Cognition between Experts and Novices”, In: International Conference on Advanced Design Research and Education 2014, Singapore, pp. 156-160. http://dx.doi.org/10.3850/978-981-09-1348-9_041

Kampylis, P.G. and Valtanen, J. (2010), "Redefining Creativity - Analyzing Definitions, Collocations, and Consequences", The Journal of Creative Behavior, Vol. 44 No. 3, pp. 191-214. http://dx.doi.org/10.1002/ j.2162-6057.2010.tb01333.x

Kang, K.S.J., Chia, P.Z., Koronis, G. and Silva, A. (2018), "Exploring the use of a Full Factorial Design of Experiment to Study Design Briefs for Creative Ideation”, In: ASME-IDETC, Quebec, Canada, August 26-29. 
Koronis, G., Chia, P.Z., Kang, J., Siang, K., Silva, A., Yogiaman, C. and Raghunath, N. (2019), “An Empirical Study on the Impact of Design Brief Information on the Creativity of Design Outcomes with Consideration of Gender, and Gender Diversity", Journal of Mechanical Design, (Accepted Manuscript), pp. 1-35. http://dx.doi.org/10.1115/1.4043207

Koronis, G., Silva, A. and Kang, J. (2018), “The Impact of Design Briefs on Creativity: A Study on Measuring Student Designers Outcomes”, In: Marjanović D., Štorga M., Škec S., Bojčetić N., and Pavković N. (Ed.), 15th International Design Conference, Dubrovnik, Croatia, 20-24 May, Institution of Engineering Designers, Glasgow, pp. 2461-2472. http://dx.doi.org/10.21278/idc.2018.0328

Lubart, T.I. (1998), “Creativity Across Cultures”, In: Sternberg R.J. (Ed.), Handbook of Creativity, Cambridge University Press, Cambridge, pp. 339-350.

Madni, A. (2012), "Elegant Systems Design: Creative Fusion of Simplicity and Power".

Moalosi, R., Popovic, V. and Hickling-Hudson, A. (2010), “Culture-Orientated Product Design”, International Journal of Technology and Design Education, Vol. 20 No. 2, pp. 175-190. http://dx.doi.org/10.1007/s10798-008-9069-1

Morris, M.W. and Leung, K. (2010), “Creativity East and West: Perspectives and Parallels", Management and Organization Review, Vol. 6 No. 3, pp. 313-327. http://dx.doi.org/10.1111/j.1740-8784.2010.00193.x

Nisbett, R.E. and Masuda, T. (2003), "Culture and Point of View", Proceedings of the National Academy of Sciences, Vol. 100 No. 19, pp. 11163-11170. http://dx.doi.org/10.1073/pnas.1934527100

Razzaghi, M. and Ramirez, M. (2005), “The Influence of the Designers' Own Culture on the Design Aspects of Products".

Shah, J.J., Smith, S.M. and Vargas-Hernandez, N. (2003), "Metrics for Measuring Ideation Effectiveness", Design Studies, Vol. 24 No. 2, pp. 111-134. http://dx.doi.org/10.1016/S0142-694X(02)00034-0

Shah, J.J., Vargas-Hernandez, N.O.E., Summers, J.D. and Kulkarni, S. (2001), "Collaborative Sketching (CSketch) - An Idea Generation Technique for Engineering Design”, The Journal of Creative Behavior, Vol. 35 No. 3, pp. 168-198. http://dx.doi.org/10.1002/j.2162-6057.2001.tb01045.x

Shai, O., Reich, Y., Hatchuel, A. and Subrahmanian, E. (2013), "Creativity and Scientific Discovery with Infused Design and its Analysis with C-K Theory”, Research in Engineering Design, Vol. 24 No. 2, pp. 201-214. http://dx.doi.org/10.1007/s00163-012-0137-x

Sternberg, R.J. (2006), “The Nature of Creativity”, Creativity Research Journal, Vol. 18 No. 1, pp. 87-98. http://dx.doi.org/10.1207/s15326934crj1801_10

Stoltzfus, G., Leigh Nibbelink, B., Vredenburg, D. and Hyrum, E. (2011), “Gender, Gender Role, and Creativity”, Social Behavior and Personality: An International Journal, Vol. 39 No. 3, pp. 425-432. http://dx.doi.org/10.2224/sbp.2011.39.3.425

Tomassoni, R., Treglia, E. and Tomao, M. (2018), “Creativity across Cultures: A Comparison between Ugandan and Italian Students”, Creativity Research Journal, Vol. 30 No. 1, pp. 95-103. http://dx.doi.org/10.1080/ 10400419.2018.1411565

Vaughan, M.R., Crawford, R.H. and ASEE (2013), "Use of Concept Generation Techniques in Different Cultural Settings", In: 2013 ASEE Annual Conference.

Voss, M., Sauer, T. and Bozkurt, H. (2014), "Using Design Heuristics in Idea Generation: Does it Take Expertise to Benefit?”, In: Bohemia E., Eger A., Eggink W., Kovacevic A., Parkinson B., and Wits W., (Ed.), 16th International Conference on Engineering and Product Design Education, Netherlands, 4-5 September, Institution of Engineering Designers, Glasgow, UK, pp. 574-579.

\section{ACKNOWLEDGMENTS}

This work is supported by the Singapore University of Technology and Design (SUTD) and the SUTD-MIT International Design Centre (IDC, idc.sutd.edu.sg), project IDG 11600102. The authors thank Dr. Nilanjan Raghunath, and Jacob Kang for their input. We are grateful to the participating students, faculty and teaching assistants from SUTD and the University of Lisbon. Portions of this paper's data have been previously presented at the DESIGN 2018 Conference (G. Koronis et al., "The Impact of Design Briefs on Creativity: A Study on Measuring Student Designers Outcomes"). Part of this work was developed within the frame of project: Projeto Mobilizador n. ${ }^{\circ} 24541$ - PRODUTECH SIF - Soluções para a Indústria de Futuro (Solutions for the Industry of the Future), supported by the Portuguese Agency of Innovation, whose support must be acknowledged. 